\title{
- Methods for Evaluating and Comparing the Use of Haptic Feedback in Human-Robot Interaction With Ground-Based Mobile Robots
}

\author{
Daniel J. Brooks, Katherine M. Tsui, Michael Lunderville, and \\ Holly A. Yanco \\ University of Massachusetts Lowell
}

A significant amount of research has been conducted regarding the technical aspects of haptic feedback. However, the design of effective haptic feedback behaviors for controlling ground-based mobile robots is not yet well understood from a human-robot interaction perspective. Past research of haptic feedback behaviors for mobile robots has sometimes made use of control paradigms that do not appropriately map to teleoperation or supervision tasks. Furthermore, evaluation of haptic behaviors has not been systematic and often only demonstrates feasibility. As a result, comparing various techniques is difficult. In this article, we focus on how haptic control research could be improved in the domain of teleoperation and supervision of ground-based mobile robots through the introduction of a haptic evaluation toolkit.

Keywords: Haptic control, haptic joystick, mobile robots, human-robot interaction, evaluation of haptic control

\section{Introduction}

The area of haptic feedback research is vast, even when restricted to work related to robots; a cursory search for the phrase "robot haptic feedback" in Google Scholar returns more than 37,500 results. Haptic feedback has been used to control robot arms (e.g., Park \& Khatib, 2006), wheelchairs (e.g., Luo, Hu, Chen, \& Lin, 1999; Christensen, 2011), rotary cranes (e.g., Takemoto, Yano, Miyoshi, \& Terashima, 2004), surgical equipment (e.g., Meijden \& Schijven, 2009; Okamura, 2004), and remote mobile platforms (e.g., Lee, Sukhatme, Kim, \& Park, 2005; Mullins, Horan, Fielding, \& Nahavandi, 2007; Rösch \& Schilling, 2002). Research on haptic feedback has included mechanical hardware design (e.g., Cho, Jin, Lee, \& Yao, 2010), software algorithms that simulate the feel of textures (e.g., Ruspini, Kolarov, \& Khatib, 1997), the use of exoskeletons and virtual fixtures to remotely control robot arms (e.g., Rosenberg, 1995), semiautonomous shared control behaviors, such as artificial force reflection (e.g., Nguyen \& Ryu, 2011; Lee et al., 2005), and implementing stable bilateral control systems inspired by physics concepts (e.g., Anderson \& Spong, 1989; Niemeyer \& Slotine, 1991; Satler, Avizzano, Frisoli, Tripicchio, \& Bergamasco, 2009).

This article is specifically concerned with leveraging haptic feedback to improve the task of teleoperating and supervising the navigation of ground-based mobile robots capable of various lev-

\footnotetext{
Authors retain copyright and grant the Journal of Human-Robot Interaction right of first publication with the work simultaneously licensed under a Creative Commons Attribution License that allows others to share the work with an acknowledgement of the work's authorship and initial publication in this journal.
}

Journal of Human-Robot Interaction, Vol. 4, No. 1, 2015, Pages 3-29. DOI 10.5898/JHRI.4.1.Brooks 
els of autonomous behaviors. Teleoperation (Sheridan \& Verplank, 1978) is typically accomplished using a proportional velocity control device and a live, first-person view of the robot's video feed. Although the concept seems simple, teleoperating a robot at this low level can be cognitively taxing, given the "soda straw" view (Voshell, Woods, \& Phillips, 2005). An operator's visual channel may be oversaturated, particularly in time- and safety-critical domains such as urban search and rescue; robot operators fixate on the video and ignore other components of the graphical user interface (Keyes, Micire, Drury, \& Yanco, 2010). As researchers, we are searching for ways in which haptic feedback can be used to provide additional information to an operator or improve operator control. Although a significant amount of work has been conducted regarding the technical aspects of haptic feedback, designing effective haptic feedback behaviors for controlling ground-based mobile robots is not yet well understood from a human-robot interaction (HRI) perspective. The effect haptic feedback techniques have on teleoperation and supervision tasks is still unknown, despite an abundance of literature addressing haptic control of mobile robots. In many cases, experiments performed in the literature are feasibility studies that demonstrate a system performing in its intended manner as the authors describe earlier in their paper, such as in the cases of Diolaiti and Melchiorri (2003), Schill, Mahony, Corke, and Cole (2008), and Cho et al. (2010). Even the most in-depth studies sometimes only include performance measures, such as time-to-task completion and the number of collisions, and are tested by relatively few participants. The way in which users understand and perceive these haptic behaviors is therefore not well understood by the research community.

In fact, this specialized research has not yet matured to the point where experiments can be repeated or results replicated by different researchers. Ruffaldi, Morris, Edmunds, Barbagli, and Pai (2006) point out this lack of verification can partially be attributed to the difficulty of replicating an individual's experience using haptic devices, due to the unstructured nature of the interaction and also to the difficulty in obtaining the required specialized hardware. Performance measures have been proposed for evaluating the devices themselves (Hayward \& Astley, 1996), but there are no widely accepted frameworks or standard testing methods for evaluating the effects of haptic behaviors as they relate to controlling mobile robots. Kirkpatrick and Douglas (2002) created a taxonomy of goal-oriented haptic modes (or styles of use) in an attempt to distinguish various ways in which haptic systems are used with the eventual goal of evaluating suitability of haptic interfaces for a particular application. However, control of mobile robots does not cleanly fit into their taxonomy. Samur, Wang, Spaelter, and Bleuler (2007) proposed using a series of testbeds for usability evaluation based on the classification of different levels of haptic interfaces. At one end of the classification system was a virtual environment that can be evaluated by the accuracy of the rendering algorithm, followed by physical devices and controllers that can be evaluated technically for resolution, power, passivity, etc., and finally, human users at the far end, who are evaluated using techniques from human factors, such as task performance and workload. This work is promising, but the testbeds use very primitive tasks that focus only on the haptic interaction and fail to capture the effect those interactions might have when incorporated into a larger scenario.

While the computer vision and data mining communities make use of publicly available data sets for evaluation, the lack of consistency in evaluating haptic behaviors as control methods makes it extremely difficult to draw comparisons of any kind between studies. Ruffaldi et al. (2006) discussed the concept of creating ground truth data sets for haptic algorithm verification and comparison. They constructed a pen-like sensor built out of a force/torque sensor and coordinate measuring machine to probe surfaces and generate data sets. These data sets were then compared against forces generated by haptic algorithms, based on geometric models of the probed objects. Although this is an excellent step forward for some use cases of haptic interfaces, the dynamically generated haptic forces resulting from a combination of outside forces changing the world state and instantaneous operator input make this technique impractical in our domain. 
As a result of the lack of standards and methodology, we are currently unable to compare the effectiveness of even closely related techniques. For example, basic artificial force reflection (an environmental feedback strategy in which users "feel" their surroundings by having nearby objects emit haptically rendered force) is a popular haptic feedback behavior and has been implemented and tested in various ways. Schill et al. (2008) performed this technique using optic flow rather than traditional infrared range sensors. Diolaiti and Melchiorri (2003) modeled the robot as a virtual mass and the operator input and obstacles as dampened springs. Cho et al. (2010) simply demonstrated the use of a new hardware device with this same technique. Each of these studies performed different types of feasibility tests, which, while valid, makes it difficult to discuss how they compare. Developing standardized methods of testing would not only allow us to make comparisons but also understand an individual technique's suitability for use in a particular situation or its ramifications on an operator's experience.

Further complicating the research of haptically controlled mobile robots is the use of either oneof-a-kind devices or commercially available devices that are not well suited to the task. Constructing a robust haptic device from scratch is a non-trivial task - evidenced from the focus of much research (e.g. Bae, Koo, Park, \& Kim, 2001; Cho et al., 2010; Hayward, Astley, Cruz-Hernandez, Grant, \& Robles-De-La-Torre, 2004). Some simple custom haptic devices, such as 1 degree of freedom (DOF) haptic paddles, have been replicated in educational settings (Richard, Okamura, \& Cutkosky, 1997); however this device replication has not been the case with haptic joysticks designed for research purposes.

Products such as the SensAble Phantom Omni (SensAble Technologies Inc., 2011b) and Novint Falcon (Novint Technologies Inc., 2013) provide attractive alternatives to researchers not wishing to build custom control hardware. Researchers have investigated the effects of haptic feedback with respect to mobile robots using these unmodified, commercial off-the-shelf (COTS) products (e.g., Diolaiti \& Melchiorri, 2003; Farkhatdinov \& Ryu, 2010; Mitsou, Velanas, \& Tzafestas, 2006; Schill et al., 2008). In addition to being convenient and readily available platforms, they also offer the advantage of support communities and software APIs (SensAble Technologies Inc., 2011a; Nonpolynomial Labs, 2010). The most prevalent example is the Phantom device series, which consists of a 6-DOF arm connected to a stylus that can be held in the user's hand like a pen. The Phantom devices were designed for users to touch and feel virtual objects, with a software API that ties directly into OpenGL for rendering touchable 3D scenes (SensAble Technologies Inc., 2011a). All research with this device has been performed using the stylus as the interface for steering or navigating the robot, with the operator pushing the stylus across a springy imaginary surface (Diolaiti \& Melchiorri, 2003; Farkhatdinov \& Ryu, 2010; Lee et al., 2005; Mitsou et al., 2006; Mullins et al., 2007). However, using a higher DOF device, such as a 6-DOF stylus for controlling a robot only capable of 2D movement, may not be as suitable as using a more traditional and ubiquitous 2-DOF input device, such as a joystick (Bowman et al., 2008; Lapointe, Savard, \& Vinson, 2011). Using uncommon methods for controlling a robot makes it difficult to separate haptic effect consequences from the control paradigm and to compare results using the haptic interface with non-haptic results acquired using the more ubiquitous, traditionally used joystick interface.

To address these issues related to the experimentation process surrounding haptic control of mobile robots, we introduce the design of a toolkit for investigating feedback behaviors. In Section 3, we demonstrate the use of this toolkit in a case study investigating the effects of introducing haptic feedback in a shared control scenario. We then discuss how previous work on haptic control of mobile robots could potentially benefit from the current state of our toolkit in Section 4. Finally, we look at the current limitations of our work and potential extensions and improvements based on experiments from the existing literature. 
Brooks, Tsui, Lunderville, and Yanco, Evaluating and Comparing Haptics for HRI

\section{HRI Haptics Toolkit}

Our goal is to offer a starting point for creating a suite of standardized tests to better understand how people interpret and react to the effects of haptic feedback while teleoperating a mobile robot. To understand how one aspect of a system affects the rest of the interaction, it is useful to compare a system with one or more previously tested systems commonly used for the same task. The components of human-robot interaction (including physical devices) being tested should strive to maintain as similar a form as possible to minimize the number of uncontrolled variables in such investigations. Defining generic representations for particular types of tasks that can be used for measurement purposes is also helpful.

\subsection{Joystick Controls}

In the case of controlling ground-based mobile robots, joysticks provide particularly good affordance, since the degrees of freedom presented by a joystick are easily mapped to the translation and rotation movements of many robot platforms. High DOF devices can be difficult to use for controlling mobile robots, especially when all axes have been mapped to various actions. Steering wheels are also usually suboptimal, due to the fact they allow only a single degree of freedom, and most people already associate this control paradigm with Ackerman (car-like) steering. Many mobile robot platforms are built to use differential (skid) or holonomic drives, which allow for behaviors that can be much more complex than the motion usually allowed by a steering wheel. Finally, joysticks also provide mechanical haptic feedback (meaning that the user is aware of the position in which he or she is holding the device) provided by a spring loaded centering mechanism and the presence of a physical bezel that limits the device's movement. For these reasons, the use of joystick style controls is strongly encouraged.

We also advocate for the use of the Robot Operating System (ROS) (Quigley et al., 2009) in designing haptic systems. ROS allows roboticists to share their software in such a way that it can be easily reused by others. This reuse has led to the creation of standards for interfacing with hardware and algorithms, allowing researchers to easily switch between components capable of providing the same service. The standard version of ROS does not provide real-time guarantees that can lead to instability, which is especially problematic for bilateral control. However, we have found that, in practice, such systems will remain stable provided that time critical components reside on the same host machine and are not subjected to processing delays.

2.1.1 Phantom Omni Augmentation As previously mentioned, one issue with some of the prior work includes the use of a stylus for driving a robot. These cases all made use of the Phantom Omni device, which comes with a stylus as the stock interface. Since no argument was made for the benefits of a stylus for controlling the robot that could not be attributed to the haptic effects, the stylus was most likely chosen because there was no readily available alternative.

Since many researchers have access to the Phantom Omni device, which is not suitable for controlling mobile robots in its off-the-shelf configuration, we created a 2-DOF haptic joystick system (Brooks, Lunderville, \& Yanco, 2013) specifically designed for this purpose. The augmentation was implemented such that other researchers wishing to perform joystick-related tasks could easily reproduce our design and includes software that maps the 6-DOF arm into just 2 dimensions (joystick rotation is not yet supported by our software). The joystick's range of motion was modeled after CH Product's M11L601P industrial analog joystick, which has previously been used in commercial robotics applications.

The addition of the joystick adapter made programming the Phantom Omni using SensAble's APIs difficult, resulting in the development of a $2 \mathrm{D}$ joystick interface and calibration routine. The joystick's 2D position and forces were exposed through ROS topics (see Fig. 1), which by default 


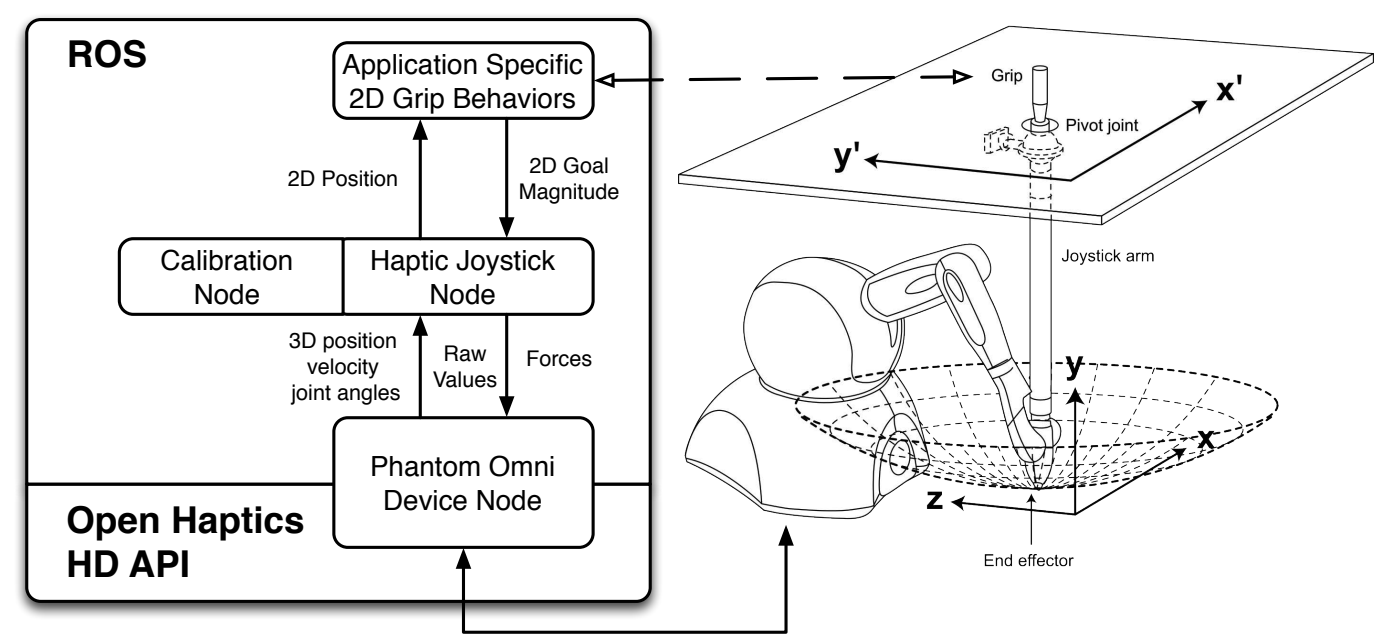

Figure 1 : Architecture Block Diagram of the Haptic Joystick Software

emulates the spring centering forces of a mechanical joystick. Application specific joystick behaviors are created by translating the location of the joystick's centering motion and the stiffness of the spring force. The joystick adapter and ROS interface have been tested by paid participants for over 60 hours of use with almost no instances of instability. The only exceptions occurred during periods of significant network latencies in excess of $200 \mathrm{~ms}$ delays while running over a wireless network.

\subsection{Experiment Design Template}

As we have already asserted, it is very difficult to compare the effectiveness of various haptic behaviors from the literature. One of the major contributing factors to this difficulty is the lack of consistent methods for testing. We do not attempt to define a one-size-fits-all experiment; rather, we propose a starting point for those studying the control of mobile robots.

In this section, we describe an experiment template suitable for conducting a wide range of tests for haptic teleoperation and supervision feedback behaviors that incorporate techniques used in human factors and human-robot interaction. The basic experimental design consists of a withinsubjects study in which an operator's primary duty is to drive, or supervise in the case of an autonomous system, a remote mobile robot through a slalom course using two different control methods. This generic primary task has been used successfully in previous experiments to investigate various factors relating to remote teleoperation at two universities ${ }^{1}$ (see Desai, 2012; Desai et al., 2012; Desai, Kaniarasu, Medvedev, Steinfeld, \& Yanco, 2013). In addition to the primary task of driving or navigating the robot, secondary tasks can be integrated based on research goals. For example, the operator could be asked to find something hidden along the course or perform some kind of delivery or manipulation task.

The primary task has been carefully designed to mitigate the effects of participants learning patterns in the task during each session and to balance out any biasing effects that might result from running both experimental conditions with each participant. Each participant performs two trial runs, followed by six runs during which data can be recorded. The two control methods (conditions) are counterbalanced for the starting condition and then alternated between the six runs. Eighteen unique sets of participant conditions are used to eliminate any potential biasing by balancing the order of

\footnotetext{
${ }^{1}$ Carnegie Mellon University and the University of Massachusetts Lowell
} 


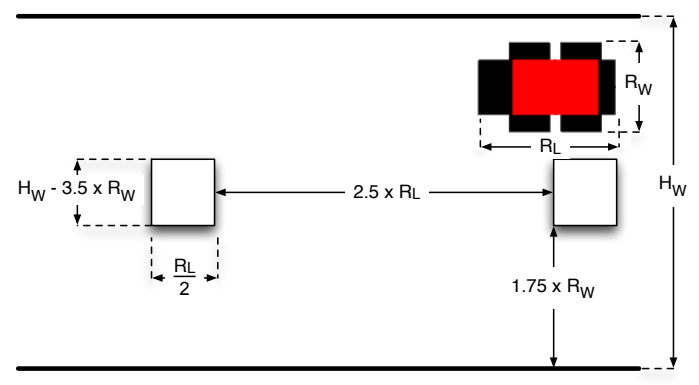

Figure 2 : Course Layout Parameters. $R_{L}$ : robot length, $R_{W}$ : robot width, $H_{W}$ : hall width

the six maps and three event sequence variations. Each set of participant conditions consists of a unique ordering of the maps when paired with the map variations. Each map is used exactly once, and each event sequence variation is used exactly twice for each participant. Whenever possible, the same physical control devices and graphical user interface should be used in both control modes, thus making the only difference the haptic effects being tested. In Section 4, we discuss how this experimental methodology could have been used in a variety of haptic experiments.

Finally, the experiment's testing environment has been designed to be accessible for a wide range of research groups. Different groups have access to different resources, including operating budgets and space available for performing testing. Our environment has been designed to be low cost and constructed in many readily available spaces. While simulators offer an attractive alternative to performing tests with physical platforms, they are not necessarily the best option for HRI experimentation. There has been relatively little work in the way of validating the use of simulators for HRI. Lewis, Wang, and Hughes (2007), in their validation for USARSim, asserted that high quality graphics and accurate physics are required for the use of simulation in HRI research-a standard that not all simulators can meet. Additionally, simulators must accurately reflect sensor values and the hardware behavior characteristics of the devices being used inside the simulation, all of which need to be validated against their physical equivalent. Shortcomings in any of these areas can have direct effects on the observed human-robot interaction. Finally, some aspects of robot operation are simply difficult to capture in simulation, such as the need to exercise caution when driving at high speeds. To date, many HRI researchers continue to use physical experiments. Because of this, our environment was designed to be physically built at low cost, easily constructible, and can be located in many readily available spaces.

2.2.1 Course Layout The testing environment was designed to be simple and easily reproducible. The result is a course consisting of a series of eight obstacles placed at regular intervals lengthwise down the center of a straight and wide hallway area. The driving task consists of guiding the robot down the hallway, making a u-turn, and returning to the starting point, while weaving between the obstacles as in a slalom course.

The course can be configured to match the size of the robot being operated (see Fig. 2). The spacing between obstacles should be approximately 2.5 times the length of the robot, and each obstacle's length should be approximately half the total length of the robot. Obstacles' widths are determined by the width of the hallway and the width of the robot, with the summed amount of space on either side of the robot as it passes by an obstacle approximately equal to $75 \%$ of the robot's width.

While this configuration allows for plenty of space to maneuver the robot, prior experimentation indicated that manually navigating the obstacles is a non-trivial task (Desai, 2012). The course can be constructed from any material; however, we suggest using cardboard or plywood. These 
materials are easy to set up, can be stored compactly, and cause minimum damage to a robot if a collision occurs at high speeds. False walls can also be built, rather than using the walls of a hallway.

2.2.2 Data Collection There are a number of important components that should be measured when comparing control methods: the participant's spatial reasoning ability, workload and ability to maintain situation awareness during a run, task performance, and perception of performance. Standardizing variables and methods of measuring them will allow us to begin establishing comparisons between experiments. However, data collection should not be limited to the methods described in this section, and additional task specific measures should be included whenever appropriate.

Spatial Reasoning. A person's ability to control a remotely located robot is linked with their spatial reasoning (SR) capability (Lathan \& Tracey, 2002). An operator with well-developed SR ability will find the task of operating a remote robot easier than someone with low ability in this area. The ETS Cube Comparison test (Ekstrom, French, \& Harman, 1979) is used for measuring participants' SR ability. This test only needs to be performed once for each participant and can be administered before or after the experiment.

Workload. The NASA Task Load Index (TLX) (Hart \& Staveland, 1988) is a subjective measurement of a participant's perceived workload. The TLX questionnaire asks the participant to rate (on a scale of 0 to 20, with 11 equaling neutral) their Mental Demand, Physical Demand, Temporal Demand, Performance, Effort, and Frustration for an activity. The TLX survey should be administered following each use of the robot. A free online TLX survey is available at www.playgraph.com/nasatlx (Sharek, 2011, 2013) and methods for analyzing the survey can be found in Hart (2006).

Situation Awareness. Situation Awareness (SA) is defined as "The perception of the elements in the environment within a volume of time and space, the comprehension of their meaning, and the projection of their status in the near future." (Endsley, 1988) There are three levels of SA: perception (level 1), comprehension (level 2), and projection (level 3). SA can be measured using the Situation Awareness Global Assessment Technique (SAGAT), which was originally developed for measuring SA in aircraft pilots (Endsley, 1988). The technique consists of periodically pausing an experiment or simulation, blacking out the interface, and asking the operator a series of questions related to the current situation four times during each run. Questions should span all three levels of SA, and there should be enough questions such that a subset can be selected at random from a pool for each SAGAT screen. Tables 1 and 2 provide a base of generic SA questions; there are nine multiple choice questions (three SA Level 1 questions, five SA Level 2 questions, and one SA Level 3 question) and two additional map-based, multidimensional questions. One multidimensional question asks the participant to select the location of the box nearest the robot from 14 discrete positions (see Fig. 3a; SA level 1 or 2), and the other requires the participant to select the position and orientation of the robot on the course (see Fig. 3b; SA level 2). A question is classified as SA Level 1 (perception) versus SA Level 2 (comprehension) if it could be correctly answered by looking at the operator interface, if it was not hidden from view. The distribution of SA Levels among questions is not special, and additional questions should be added based on customizations that have been made to the experiment.

Task Performance. A number of metrics directly related to the task should be recorded for each run. These metrics are useful as indirect measures of SA and the usability of an interface (Steinfeld et al., 2006). Task performance metrics include:

- Time to completion: Time elapsed if the task was successfully completed. 
Brooks, Tsui, Lunderville, and Yanco, Evaluating and Comparing Haptics for HRI

Table 1: Single Dimension SAGAT Questions. Response options are “yes," “no,” and "I don’t know.”

\begin{tabular}{|c|c|l|l|}
\hline Num & $\begin{array}{c}\text { SA } \\
\text { Level }\end{array}$ & Question & Restrictions and Notes \\
\hline \hline 1 & 1 & $\begin{array}{l}\text { When the screen went black, were you driving the robot at } \\
\text { full speed? }\end{array}$ & $\begin{array}{l}\text { Full speed is defined as the joystick stick } \\
\text { being pushed all the way in any direc- } \\
\text { tion. }\end{array}$ \\
\hline 2 & 1 & Have you passed the u-turn yet? & $\begin{array}{l}\text { Must not be asked just after the begin- } \\
\text { ning of the run, just before the end of } \\
\text { the run, or just before the u-turn. }\end{array}$ \\
\hline 3 & 1 & $\begin{array}{l}\text { Are there less than four boxes remaining in the direction } \\
\text { you are currently headed? }\end{array}$ & $\begin{array}{l}\text { Must not be asked just after the begin- } \\
\text { ning of the run, just before the end of } \\
\text { the run, or just before the u-turn. }\end{array}$ \\
\hline 5 & 2 & On what side did the robot pass the last box? & - \\
\hline 6 & 2 & $\begin{array}{l}\text { On what side did the robot pass the second to last box? } \\
\text { robot crossed the line of boxes down the middle of the } \\
\text { hallway? }\end{array}$ & $\begin{array}{l}\text { Must not be asked on the first screen af- } \\
\text { ter the u-turn (could cause ambiguity). }\end{array}$ \\
\hline 7 & 2 & $\begin{array}{l}\text { Since [the start of the run/the last black screen], has the } \\
\text { robot bumped or scraped into a box or wall ? }\end{array}$ & $\begin{array}{l}\text { - } \\
\text { passed any boxes on the wrong side? }\end{array}$ \\
\hline 9 & 2 & $\begin{array}{l}\text { Since [the start of the run/the last black screen], have you } \\
\text { Would it be safe to drive the robot forward at full speed } \\
\text { for the next two seconds? }\end{array}$ & - \\
\hline
\end{tabular}

- Number of collisions: Number of occurrences in which the robot bumped, scraped, or hit any part of the environment.

- Number of mistakes: Number of occurrences in which mistakes are made. For the primary task this means making a wrong turn. Secondary task mistakes should also be counted here.

Subjective Measures. Finally, a number of subjective measures can be recorded. These should be asked after each run and can be administered along side the TLX questionnaire. Other experimentspecific questions can also be added to this set:

- Which condition did you prefer?

- Rate how predictable the system was during the last run (1=Unpredictable to $10=$ Very Predictable)

- Rate the robot's performance during the last run ( $1=$ Very Poorly to $10=$ Very Well $)$

- Rate how well you feel you performed during the last run (1=Very Poorly to 10=Very Well)

2.2.3 Maps and Event Sequences. A total of seven maps (six plus the Trial map) and four event sequences that are applied to the maps (three plus the trial variation) are needed to provide each participant with a unique experience. Event sequences are combinations of planned mistakes (discussed later in this section) and SAGAT screen interruptions that have been designed to have the same level of task difficulty for each run.

Maps. For each map, the robot should pass a total of 15 obstacles ( 7 boxes out, u-turn, and 7 boxes back), such as shown in Fig. 4. Each map has been designed to have the robot "crossing" the line of boxes a total of six times, not including moving to or from the starting point to the first box or going around the u-turn. Crossings should occur no less than twice and no more than four times in any given direction (out or back). Additionally, maps need to take into account the locations of planned mistakes in each event sequence variation. Alternate maps could be used, but care should be taken to balance map configurations as described here, and new event sequences would need to be generated as described in the following sections. 
Brooks, Tsui, Lunderville, and Yanco, Evaluating and Comparing Haptics for HRI

Table 2: Multidimensional SAGAT Questions

\begin{tabular}{|c|c|c|c|}
\hline Num & $\begin{array}{c}\text { SA } \\
\text { Level }\end{array}$ & Question & Restrictions and Notes \\
\hline 10 & 1 or 2 & $\begin{array}{l}\text { Please click the area that best represents the location of the } \\
\text { box nearest to the robot, keeping in mind that the closest } \\
\text { box may be behind you. The square in the center repre- } \\
\text { sents the robot. } \\
\text { Additional Verbal instructions: Rows } 1 \text { and } 5 \text { represent } \\
\text { the closest box being at least one full robot length away. } \\
\text { L3 and L5 represent the closest box being directly beside } \\
\text { the robot. }\end{array}$ & $\begin{array}{l}\text { See Fig. 3a. There exist configurations } \\
\text { in which being able to see the display } \\
\text { would not help to answer the question; } \\
\text { for example, if a blind spot exists } \\
\text { in the robot's sensors (i.e., camera } \\
\text { views, distance ranging information), } \\
\text { then this question is considered Level 2. }\end{array}$ \\
\hline $10 \mathrm{a}$ & & Is the letter $[\mathrm{L}, \mathrm{F}, \mathrm{R}$, or $\mathrm{B}]$ the same? & Graded as "yes" or "no" \\
\hline $10 \mathrm{~b}$ & & Is the row number the same? & Graded as "yes" or "no" \\
\hline $10 \mathrm{c}$ & & $\begin{array}{l}\text { Is the answer with one Manhattan distance of the correct } \\
\text { answer? }\end{array}$ & Graded as "yes" or "no" \\
\hline 11 & 2 & $\begin{array}{l}\text { First click on the map the area that best represents the } \\
\text { robot's position. Then click on the arrow that best rep- } \\
\text { resents the robot's current direction. }\end{array}$ & See Fig. $3 b$ \\
\hline $11 \mathrm{a}$ & & $\begin{array}{l}\text { Is the selected location located within the same row (Left, } \\
\text { Center, or Right) as the correct answer? }\end{array}$ & Graded as "yes" or "no" \\
\hline $11 \mathrm{~b}$ & & $\begin{array}{l}\text { Is the selected location within the same column (1-17) as } \\
\text { the correct location? }\end{array}$ & Graded as "yes" or "no" \\
\hline $11 \mathrm{c}$ & & Is the selected direction the same as the correct direction? & Graded as "yes" or "no" \\
\hline
\end{tabular}

Planned Mistakes. A common problem in autonomous systems is operators' poor ability to recover control or correct autonomous behaviors when errors occur. This issue, known as the "outof-the-loop" problem, results from low SA (Kaber \& Endsley, 1997) and has been one of the barriers to the wider adoption of autonomous mobile robot systems. We have designed the experiment to incorporate planned failures during each run in order to study an operator's ability to recover when errors occur.

With the exception of the trial variation, each variation consists of two points along the course where the robot should make a mistake (the trial has only one planned mistake). The number of mistakes was selected to be infrequent enough to maintain the operator's trust in the robot's competence (Desai, 2012). The first mistake happens while passing an obstacle on the way out to the u-turn, and the second on the way back to the start. Each mistake happens in a unique location-in other words, there are six unique places where the robot can make the intentional errors.

What constitutes a mistake or error can be varied to represent different types of failures related to driving and/or navigation. Examples include (but are not limited to) accidental collisions, taking the wrong path, or reporting incorrect information about location.

SAGAT Screen Requirements. The location of SAGAT screen events takes into account each screen's proximity to mistake locations. There are an equal number of SAGAT screens in each direction, and, in each direction, there is one SAGAT screen for which the robot makes an error, and one for which it does not. Half of the SAGAT screens occur between boxes, and the other half occur when the robot is directly beside a box in the act of passing. For each box (except the first and the u-turn), there is exactly one instance where a SAGAT screen occurs beside it. When pairing the 'error(Y)/no-error(N)' and between 'boxes(B)/beside a box(S)' scenarios, there is one of each in each of the event sequence variations (see Fig.5).

During the two trial runs, only one SAGAT screen should be displayed. This maximizes the amount of continuous driving time the participant receives to allow for becoming familiar with the task, while also gaining familiarity with the SAGAT screens. Because there is only one SAGAT 


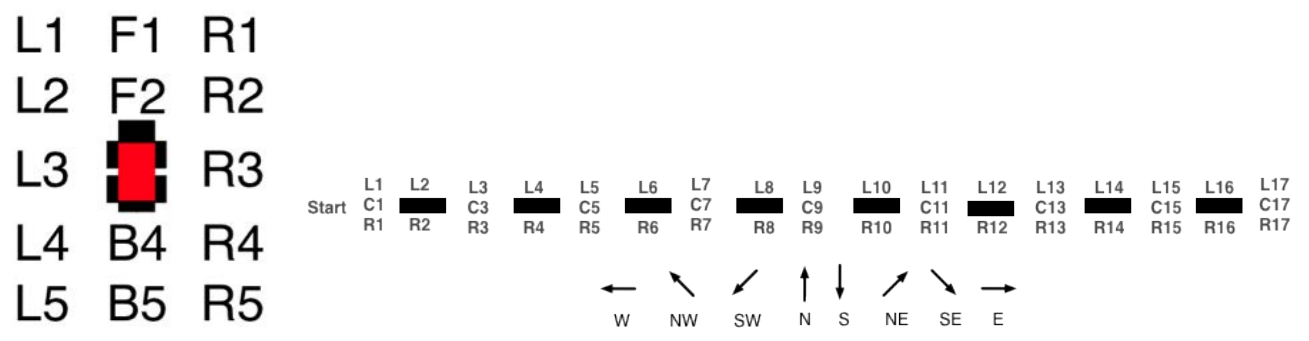

(a) Click on the area that best represents the

(b) First click on the map the area that best represents the robot's position. Then click on the location of the box nearest to the robot. arrow that best represents the robot's current direction.

Figure 3 : Multidimensional SAGAT Questions 10 and 11 in Table 2

screen in each trial run, additional questions from the pool of possible questions may be asked to help expose the participant to the types of questions to which they will respond.

2.2.4 Graphical User Interface To facilitate comparing results between different experiments, the visual components of the interface should be kept as basic and minimalistic as possible. We make the following recommendations based on prior work informing graphical user interface (GUI) design for teleoperation of remote robots (Keyes et al., 2010). The GUI should consist of a forward facing main video feed, a rear video feed, and distance display (see Fig. 6). The main video feed should be featured as the primary component in the center of the interface. An optional widget may be added to control movement of the main video feed using a pan/tilt unit. The rear video feed should be in a smaller window placed outside the top right or left hand corner of the main video feed, analogous to the position of a rear view mirror in a vehicle, varied based on the standard in the country in which the testing is occurring. The rear video stream may also be mirrored to enhance this effect. Below

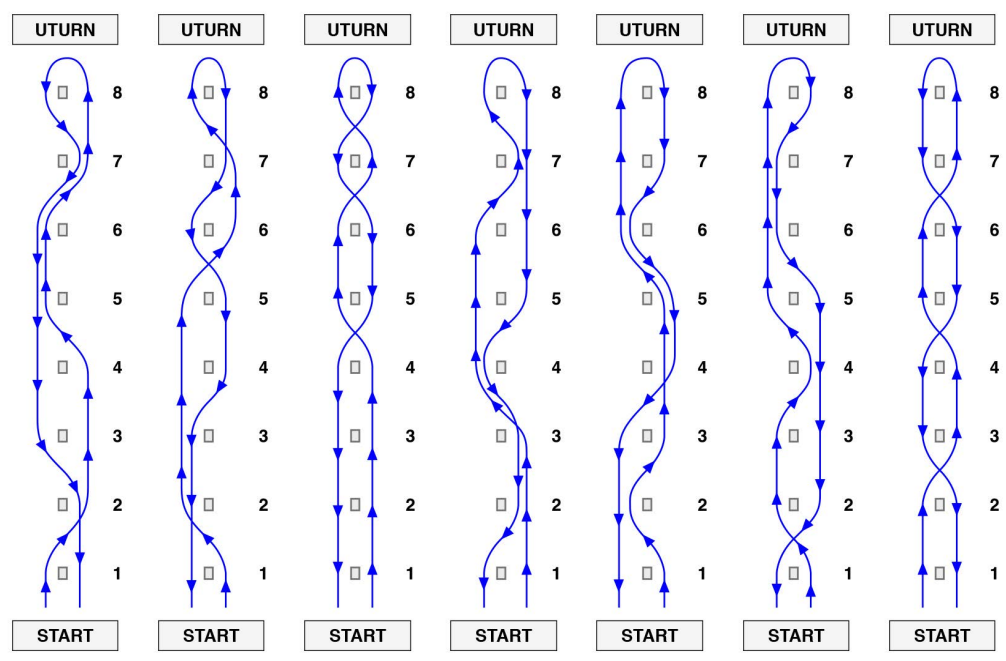

Figure 4 : Course Maps 


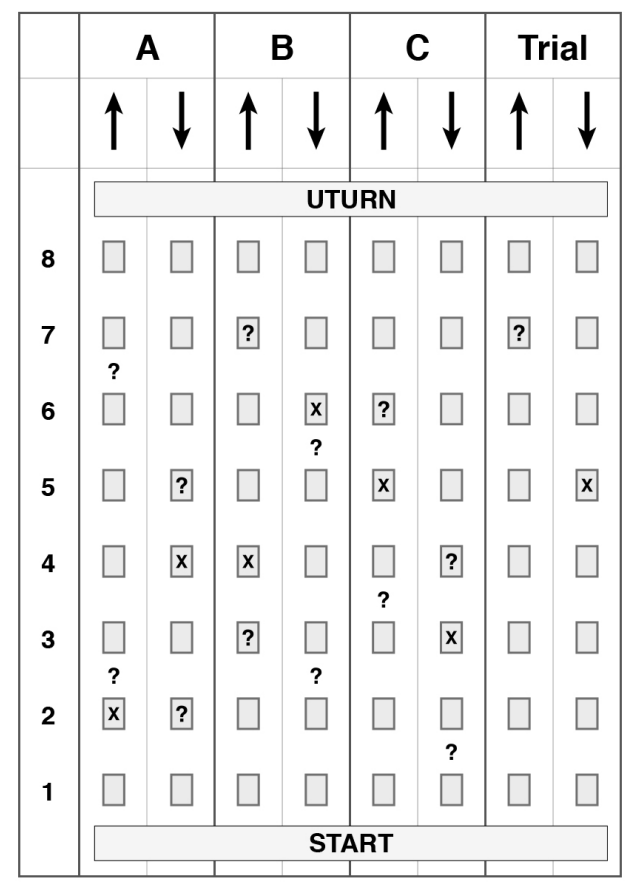

\begin{tabular}{|c|c|c|c|c|}
\hline Variation & S1 & S2 & S3 & S4 \\
\hline \hline A & YB & NB & NS & YS \\
\hline B & NS & YS & YB & NB \\
\hline C & NB & YS & NS & YB \\
\hline
\end{tabular}

Figure 5 : Event Sequence Variations. Left: Event Positions for A, B, C, and Trial. '?' denotes SAGAT screen locations, and ' $\times$ ' denotes planned mistakes. Variations were applied to maps. Right: Error and Placement Pairings. (Y) Error, (N) No Error, (B) Between Boxes, (S) Beside a Box

the main video feed should be a distance display featuring a top down view of the robot (shown as an icon), and a line drawing representing information from the robot's distance sensors (e.g., laser range finders, sonar ring). The distance display should be drawn to scale, and as complete a 360 degree view as possible. Finally, the GUI should have a small runtime clock displaying elapsed time since the beginning of the run. Additional information may be added to the GUI relating specifically to the haptic effects or any secondary tasks added to the experiment, but should be kept to a minimum.

\section{Toolkit Case Study}

Using this toolkit, we carried out an experiment investigating the effects of implementing shared control autonomy with haptic feedback on an operator's SA. This case study also demonstrates the implementation of a novel navigation system, which uses the Phantom Omni augmentation introduced in Section 2.1.1 of this paper. The independent variable in the experiment was the presence or absence of haptic feedback in the interface, referred to as "Force Feedback" and "Non-Force Feedback," respectively. In both cases, the robot was able to navigate itself through the course autonomously but could be overridden by user input. Our hypothesis was that haptic interfaces can be leveraged to help improve operator situation awareness. Specifically, we hypothesized that:

- Users will have better task performance when operating the robot with the haptic joystick.

- Users will have better SA when operating the robot with the haptic joystick.

- Users will have lower cognitive workload when using the haptic joystick.

The robot used for this experiment was an iRobot ATRV Jr platform measuring $63.5 \mathrm{~cm}$ wide x $101.6 \mathrm{~cm}$ long. According to Section 2.2.1, our obstacles needed to be $21.6 \mathrm{~cm}$ wide to provide 


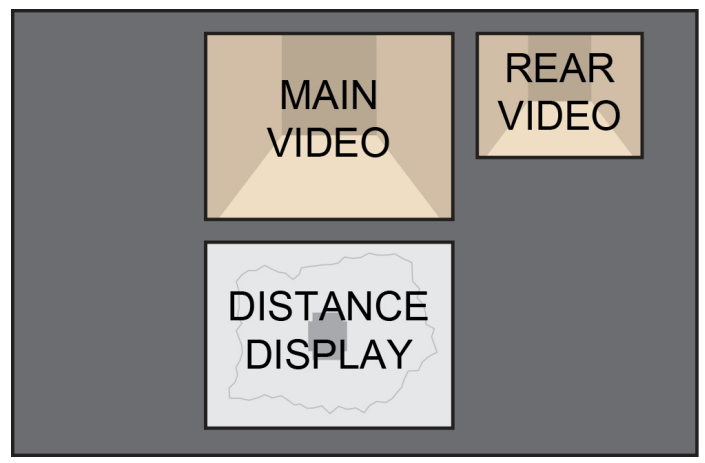

Figure 6 : GUI Layout

approximately $23 \mathrm{~cm}$ of clearance on either side of the robot in our $2.5 \mathrm{~m}$ wide hallways, and $51 \mathrm{~cm}$ long spaced $254 \mathrm{~cm}$ apart. Arrows on the boxes pointed toward which side the robot should pass each obstacle. To account for the robot's potential to make a mistake, participants were told that the robot would scan reflective barcodes placed near the bottom of each box to decide what direction to go, but that the system was not completely accurate.

The robot carried two laser ranger finders, which provided a combined 360 degrees of coverage. However, the positioning of the devices created a blind spot on either side of the chassis such that objects closer than $45 \mathrm{~cm}$ to the front wheels could not be seen. Because the blind spot only extended $30 \mathrm{~cm}$ out before the laser coverage began, this did not limit the performance of autonomous navigation. The robot also carried two cameras, a forward facing camera mounted on a pan-tilt unit near the front of the robot, and a backward facing camera near the rear of the robot.

\subsection{Autonomous Navigation}

The goal in designing the navigation system was to make the two driving conditions as similar as possible, with the only major distinguishing factor being the presence or absence, respectively, of haptic forces. We thus created an autonomous navigation behavior that could be implemented either with or without haptic effects (see Fig. 7). In the non-haptic mode, the motors were directly controlled by the autonomous software, with speed controlled by pushing the joystick directly forward along the forward-backward axis. The autonomy could be overridden by moving the joystick off-center along the left-right axis or backward along the forward-backward axis. In haptic mode, the motors were directly controlled by the position of the joystick; the autonomous software drove by invoking joystick movement (the haptic effect) and could be overridden by the operator pressing forcefully on the joystick. These behaviors are discussed in more detail in Section 3.2.3.

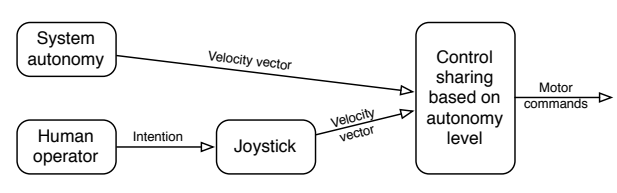

(a) Non-Haptic Shared Control

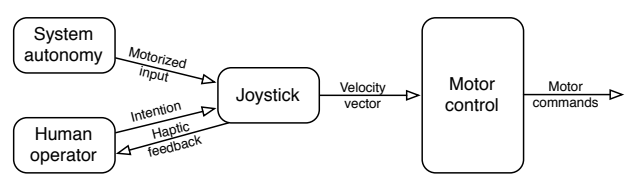

(b) Haptic Shared Control

Figure 7 : Shared Control Modes 


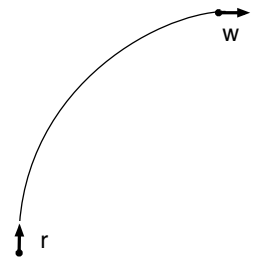

(a) Symmetric

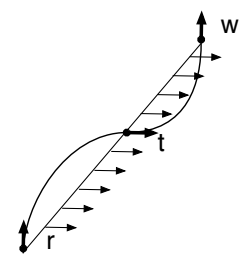

(b) Non-Symmetric, Parallel

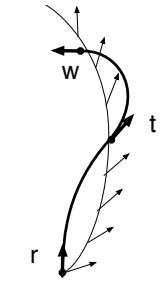

(c)

Non-Symmetric, Non-Parallel

Figure 8 : Waypose Possibilities

3.1.1 Waypose Path Planning Because the operator and the autonomy share control of the vehicle, we wanted to avoid autonomous behaviors that would feel counterintuitive to the human. The navigation system was therefore comprised of a waypose global planner and a local planning system, which created smooth trajectories between goal points and provided obstacle avoidance behaviors. A waypose differs from a waypoint by adding an orientation component to the $(x, y)$ position and threshold radius. In other words, when the robot arrived at a position satisfying the positional condition, it must have also simultaneously met the orientation requirement in order to have "arrived" at the waypose.

We defined the robot's path as a list of wayposes located at fixed points on the sides of the obstacles used in the experiment. Two sets of four wayposes were defined for each obstacle, at each of its four corners. Both waypose sets were oriented to point straight down (or parallel to) the hallway, with one set pointed away from the start and the other toward it. The path along which the robot should travel was selected as the left or right pairs of wayposes for all obstacles from the start to the u-turn and back.

Since our path was composed of wayposes rather than waypoints, conventional path planning algorithms were not sufficient because they did not consider the robot's goal orientation during the planning stage. To achieve smooth driving, we implemented a "two step" arc planning algorithm based on Kanayama and Hartman (1989).

The algorithm attempts to find a path defined by a circular arc that is tangent to both the robot's current pose and the target (or next) waypose within some threshold. These two poses are considered to be symmetric if such a path can be found that does not contain obstacles currently within view of the robot's sensors (see Fig. 8a). Otherwise, the two poses are considered non-symmetric, and an intermediate waypose must be added (Figs. 8b and 8c). This new temporary waypose $\vec{t}$ is inserted between the robot and target waypose such that it is symmetric to both of them. The temporary waypose acts as the target waypose until it either ceases to be symmetric with the robot, or the robot arrives at it. At that point, the original target waypose is rechecked to see if the robot is symmetric, and this process is repeated until the target waypose is achieved. Once this happens, a new waypose is taken from the list defining the robot's path and set as the new target waypose; this process is repeated until the list is empty.

When a temporary waypose must be placed, the next step is to determine if the robot's current pose $\vec{r}=<r_{x}, r_{y}, r_{\theta}>$ and the target waypose $\vec{w}=<w_{x}, w_{y}, w_{\theta}>$ are parallel. The two poses, $\vec{r}$ and $\vec{w}$, are considered to be parallel if $\Delta_{\text {threshold }}>\left|r_{\theta}-w_{\theta}\right|$ where $r_{\theta}$ and $w_{\theta}$ are the orientation components of $\vec{r}$ and $\vec{w}$, respectively. For cases in which the two poses are parallel, the new temporary waypose $\vec{t}$ will have an orientation $t_{\theta}=2 * \operatorname{atan} 2\left(w_{y}-r_{y}, w_{x}-r_{x}\right)-\left(r_{\theta}+w_{\theta}\right) / 2$. Its 
position $\left(t_{x}, t_{y}\right)$ will lie somewhere on the line segment defined by the points $\left(r_{x}, r_{y}\right)$ and $\left(w_{x}, w_{y}\right)$. A set of six evenly spaced discrete points along this line segment are selected as candidate temporary wayposes, $c t=\left\{\overrightarrow{c_{i}} t \mid i \in \mathbb{Z}, 0 \leqslant i<6\right\}$ (see Fig. 8b).

If the two poses, $\vec{r}$ and $\vec{w}$, are not parallel, then the set of candidate temporary wayposes, $c t$, will lie along the edge of a circle on which both the current pose and the next waypose also lie (see Fig. 8c). This circle is defined by Eq. 1. Each candidate temporary waypose's orientation is then defined as a point along the edge of the circle as described in Eq. 2.

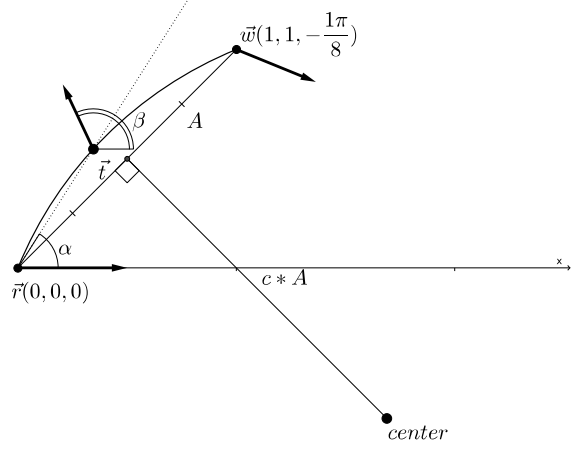

$$
\begin{aligned}
& c=\cot \left(w_{\theta}-r_{\theta}\right) \\
& \text { center }_{x}=\frac{r_{x}+w_{x}+c *\left(r_{y}-w_{y}\right)}{2} \\
& \text { center }_{y}=\frac{r_{y}+w_{y}+c *\left(w_{x}-r_{x}\right)}{2} \\
& \text { radius }=\mid \text { center }_{(x, y)}-r_{(x, y)} \mid \\
& c_{i} t_{\theta}=2 * \operatorname{atan} 2\left(c_{i} t_{y}-r_{y}, c_{i} t_{x}-r_{x}\right)-r_{\theta}
\end{aligned}
$$

Each candidate element in the set of $\left\{\overrightarrow{c_{i} t} \mid i \in \mathbb{Z}, 0 \leqslant i<6\right\}$ wayposes is tested to see if any obstacles lie along the path defined by the two arcs tangent to the candidate waypose (i.e., from the robot's current pose $\vec{r}$ to the next target waypose $\vec{w}$ ). The first candidate that produces a collision-free path for the robot to follow is selected as the temporary waypose $\vec{t}$.

3.1.2 Obstacle Avoidance and Local Planning Since the robot's autonomy does not always have direct control over the motors, we refer to the linear and angular velocities that would normally constitute driving commands as the autonomy's intention. The intention is calculated to match the arc that is tangent to both the robot and the next waypose. In the event that the robot will collide with an obstacle while traveling along this direct path before it reaches the waypose $\vec{w}^{\prime}$, we calculate the distance from the point of impact $\left(p_{x}, p_{y}\right)$ to waypose $\left(w_{x}^{\prime}, w_{y}^{\prime}\right)$ and store this value as $d_{0}=\left(p_{x}, p_{y}\right)-\left(w_{x}^{\prime}, w_{y}^{\prime}\right)$. We next test several pre-defined paths defined by arcs with radii $r=$ $\pm[0.5 \mathrm{~m}, 1.0 \mathrm{~m}, 1.5 \mathrm{~m}, 2.5 \mathrm{~m}, 4.5 \mathrm{~m}, 34.5 \mathrm{~m}]$. For each arc $a$ defined by a radius in $r$, we calculate the closest distance $d_{a}$ as the shortest distance the arc $a$ comes within reach of the waypose $\vec{w}^{\prime}$ before a collision occurs. In the case where an arc $a$ passes by the waypose $\overrightarrow{w^{\prime}}$ before a collision, $d_{a}$ is equal to the shortest distance from the waypose to arc. We then compare the distances calculated for each arc (including the original arc) and select the arc that brings us closest to the waypose as $\min \left(d_{0}, d_{a 0}, d_{a 1}, \ldots\right)$ (see Fig. 9).

\subsection{User Interface}

The operator's controls consisted of a graphical interface and physical control panel as shown in Fig. 10. The control station was located in a different area of the building from where the robot was driving, such that the operator could not see or hear the robot.

3.2.1 Graphical Interface The graphical user interface (GUI) in our experiment was based on an existing interface used in our prior research (Desai, 2012) that met our toolkit guidelines. The GUI was used in all experimental conditions and consisted of a main video feed, rear video feed, and 

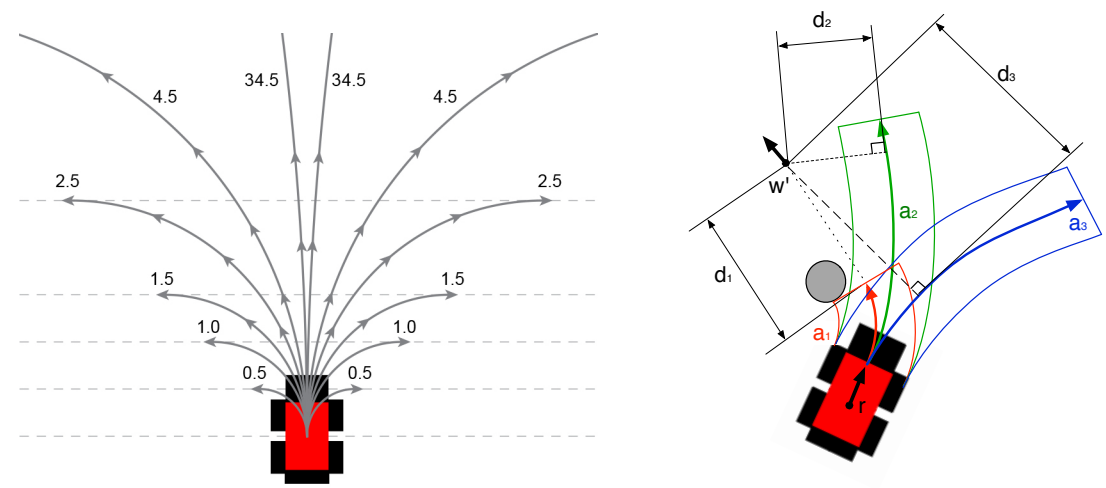

Figure 9 : Obstacle Avoidance. Left: Several arcs are calculated. Right: The arc that comes closest to the waypose is selected. In this example, $a_{2}$ would be selected, because $d_{2}<d_{1}<d_{3}$.

distance display (see Fig. 10a). The main video feed featured a yellow crosshair with white lines extending out horizontally and vertically to indicate the direction in which the camera was pointed. The rear video feed was mirrored and placed at the top right of the main video window, making it like a rear view mirror in a car (Keyes, 2007). A small runtime clock in the lower right hand corner of the display showed the elapsed time since the beginning of the run.

The distance display consisted of a top down view of the area around the robot. As the main video feed was turned from side to side, the distance display also rotated to indicate the direction the camera was pointed. The distance display consisted of a scaled icon of the robot in the center and black measurement lines extending out from each of the robot's four sides. White lines indicated distance measurements in meters to obstacles near the robot as seen by the laser sensors. Two vectors, colored blue and white, originated from the center of the robot icon. The blue vector indicated the autonomous system's intent, while the white vector indicated the actual linear and angular velocity at which the robot was actually driving.

3.2.2 Physical Controls The physical controls consisted of two joysticks and two arcade-style push buttons (see Fig. 10b). The left joystick, used for driving, was our 2-DOF haptic joystick adapter for the Phantom Omni. The right joystick was a COTS joystick by $\mathrm{CH}$ Products and used for controlling the pan and tilt of the main video feed. The two buttons located in front of the right joystick were used to re-center the video camera (the green button on the right) and to indicate incorrect robot actions whenever the robot tried to drive toward the wrong side of a box (black button on the left).

3.2.3 Autonomous Steering Behaviors In the "non-haptic" condition, the joystick's haptic feedback behavior emulated a mechanical joystick's spring centering force. The autonomy was given direct control of the motors by default and would self-navigate through the course according to the pre-defined path at a minimum forward velocity (see Fig. 7a). Whenever the joystick was centered or aligned in the positive vertical direction (i.e., pushed forward), the autonomy would retain control and accept the operator's linear velocity if greater than the minimum. Moving the joystick off-center or backward would revoke control from the autonomy. Once control had been revoked, the operator was granted full position-velocity control (teleoperation). Unlike traditional teleoperation, however, returning the joystick to a centered position or moving into a forward aligned position returned control to the autonomy, which would continue to drive the robot rather than the traditional behavior 


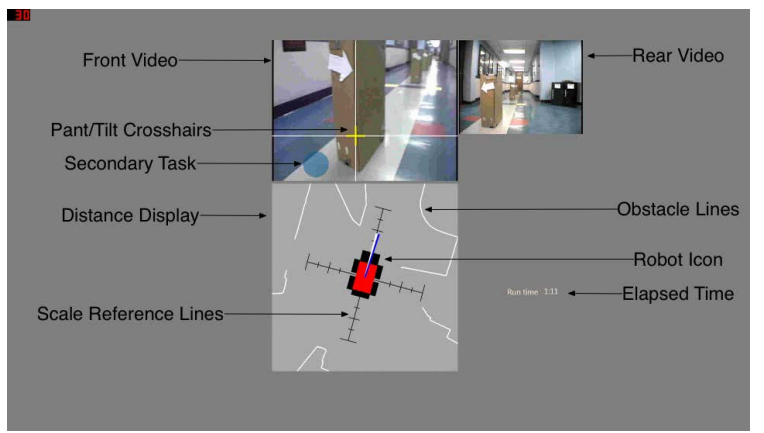

(a) Graphical User Interface

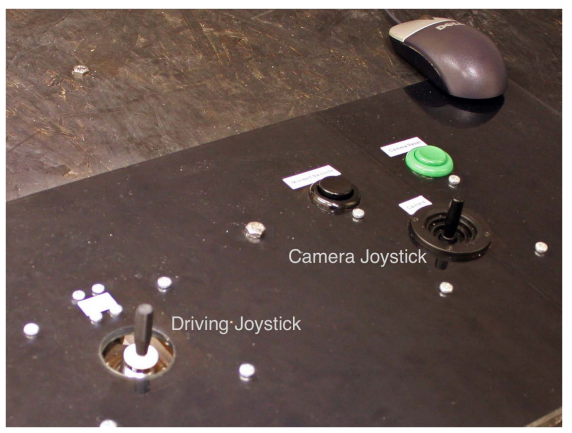

(b) Physical Control Panel

Figure 10 : Operator Control Station

of coming to a stop. The robot could be effectively stopped by pulling slightly backward on the joystick. Due to the joystick emulating a spring centering force, if the operator released the joystick, it would self-center in the same way a mechanical joystick behaves.

In the haptic condition, the joystick had absolute control over the robot's motors, and both the autonomy and the human operator used the joystick to control the robot's actions (see Fig. 7b). The autonomy was able to invoke joystick movement using the force feedback mechanism by specifying a target joystick grip location and maximum force magnitude to use in achieving the position. Autonomous steering forces were combined with a joystick centering force to create a single force effect that the operator could feel. However, unlike the non-haptic condition, if the operator let go of the joystick grip in this condition, the joystick would not re-center. Instead, it would settle into a slightly forward position (necessary to allow the autonomy to continue driving the robot forward) and occasionally shift from side to side under self-power to allow the autonomy to steer around obstacles while giving a haptic indication of the robot's intent. Since the joystick directly controlled the robot's motors, the operator could override the autonomy by pressing forcefully on the joystick grip. By pressing the joystick in a forward direction while still allowing the autonomy to move the joystick from side to side, the operator could influence the robot's speed while effectively allowing the autonomy to perform the task of steering. The haptic force applied to the joystick would increase as the actual physical joystick grip location deviated from the target position determined by the autonomy's intention (i.e., due to the operator pushing on the grip).

Although the robot was capable of autonomously navigating the entire course in either condition much faster than a human operator, the default speed of the robot when the operator was not touching the controls was set to be much slower. This was done to encourage the operator to "participate in driving" by pushing forward on the joystick to change the robot's speed while the autonomy continued steering. For safety reasons, the maximum speed was limited to match the pace a person could walk comfortably. The maximum speed the robot could be driven at and the default speed at which the autonomy would drive if the operator was not touching the controls were the same between conditions.

3.2.4 Complying with the User Since control of the robot was shared with a human operator, the autonomy could be overruled at any point; that is, the user could steer in a different direction than the autonomy's calculated intention. The reasons why the operator would choose to override the autonomy could be broken down into two scenarios, assuming that the operator wanted to drive down the hallway in the same general direction as the robot. 


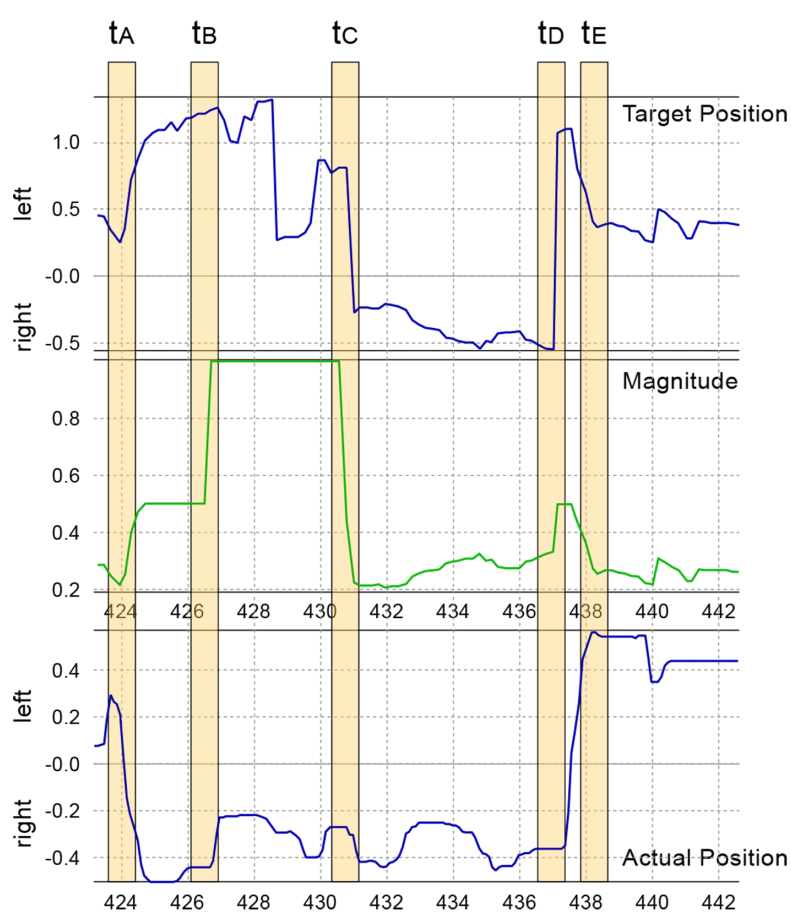

(a) Haptic Forces

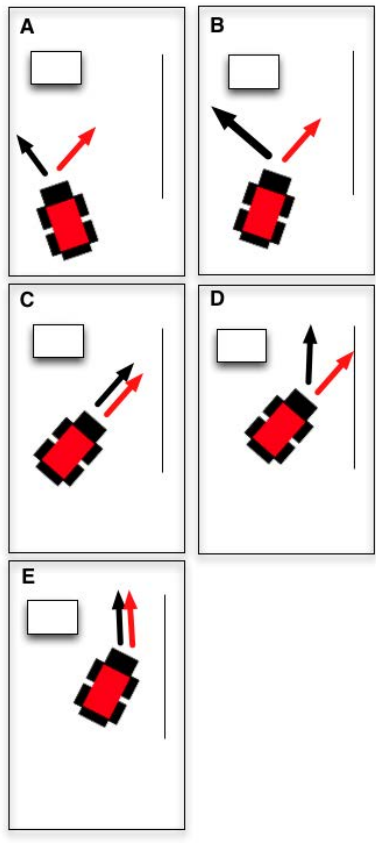

(b) Robot Location

Figure 11 : User Compliant Haptic Behavior Example. (Left) Top: Target joystick angular position, Middle: Magnitude of force $(m)$, Bottom: Actual joystick angular position. (Right) Corresponding scenarios at $t_{n}$. The black arrow indicates the robot's intention; the red arrow indicates the joystick command.

In the first scenario, the operator and autonomy both want to drive toward the same side of the next obstacle, but the operator wants to temporarily change the robot's course. Reasons might include wanting to move the robot closer or further away from the wall or making more aggressive turns than the autonomy normally makes. The result is that once the operator returns control to the autonomy, the autonomy should continue steering toward the same fixed waypose.

In the alternative scenario, the operator disagrees with the autonomy about which side the robot should pass on the next obstacle. The most likely reason for this disagreement is the planned mistakes built into the experiment. In this case, because of the disagreement between the pre-defined path the robot is following and the direction of the arrow in the remote environment, the operator will override the autonomy to steer the robot toward the opposite side of the obstacle. When the operator returns control to the autonomy, the autonomy should accept this action as a correction and modify its intention to steer toward the direction chosen by the user.

The following example demonstrates the compliant behavior of the joystick in the haptic condition (Brooks et al., 2013). The graphs in Fig. 11a were taken from data collected during our study and show the actual angular position of the joystick grip as well as the desired haptic target and force magnitude exerted over time. In this scenario, the robot was initially steered toward the left (incorrect) side of an approaching obstacle; the autonomy intentionally encouraged the behavior as a result of a planned mistake. The participant, correctly identifying the robot's mistake, began to intervene and correct the mistake by pushing the joystick to the right $\left(t_{A}\right)$. The autonomy, still believing the 
robot should be headed left, responded by increasing the magnitude of force pushing the joystick left. As the participant deviated further from the autonomy's desired direction, it increased the force pushing left again $\left(t_{B}\right)$ in an attempt to change the participant's actions. However, as the participant persisted in contradicting the autonomy, the autonomy modified its fixed waypose to comply with the participant's correction $\left(t_{C}\right)$. As the robot crossed the front of the obstacle, it became necessary to turn left to continue down the hallway. The robot again exerted a leftward force $\left(t_{D}\right)$ to which the participant complied. As the participant and autonomy both became satisfied with the rate of turn, the autonomy applied a weak centering force $\left(t_{E}\right)$ to simulate the springs of a physical joystick.

\subsection{Tasks}

The participant's primary task was to ensure the robot navigated the course following the correct path (as indicated by the arrows) while not hitting anything. Since both modes were autonomous, this task consisted of monitoring the robot's behavior and making corrections as necessary.

The areas of the course for planned mistakes were used to simulate the robot misreading a barcode telling it what direction to travel, resulting in the robot attempting to pass on the wrong side of an obstacle. These errors allowed the experimenters to see how participants reacted to failures of the autonomy, and how the absence or presence of haptic feedback influenced the event. There were two types of mistakes the robot could make. It could go straight when it should have turned, or it could turn when it should go straight. The type of mistake the robot made at a specified error point depended on which map was used. These mistakes were balanced such that each participant experienced an equal number of each type of mistake for both control methods. When the robot did make a mistake, participants were instructed to press the black button and steer the robot toward the correct side of the box.

In addition, participants were asked to perform a secondary tracking task. Translucent blue circles, 70 pixels in diameter, were generated over the main video feed (Desai, 2012). Every 35 seconds, a circle would appear in a pseudorandom location at a distance of 150 pixels away from the crosshair's current location. Whenever one of these circles appeared, participants were instructed to move the yellow crosshair over the circle so that it would disappear. This secondary task forced participants to move the front camera off-center.

Finally, participants needed to remember information relating to what was happening around the robot, such as which side the robot passed the last box on, where the robot was on the course, if the robot had made any recent mistakes, and if the robot had hit anything. Four times during each run (twice in each direction), the robot was stopped and the interface was obscured to ask the participant SAGAT questions (see Tables 1 and 2).

\subsection{Compensation}

Participants received $\$ 20$ in base compensation for completing the experiment and had the opportunity to earn an additional $\$ 20$ based on their performance. For the performance bonus, participants were told that they would start with all $\$ 20$ of the additional payment and would lose $\$ 5$ for each wrong turn the robot made, $\$ 1$ for each time the robot bumped or scraped a box or wall, or $\$ 0.50$ for each missed tracking circle (i.e. when the circle disappeared before they moved the yellow crosshair over it). They were told that these costs were averaged over all six runs and then rounded up to the nearest $\$ 5$. In order to motivate participants to do their best at maintaining situation awareness, they were also told that they would lose $\$ 0.50$ for each incorrectly answered SAGAT question; however, the actual performance bonus did not end up taking this into account due to technical reasons. Prior to being paid the compensation, participants were not given any indication of their performance level, other than what they could observe from the video. 


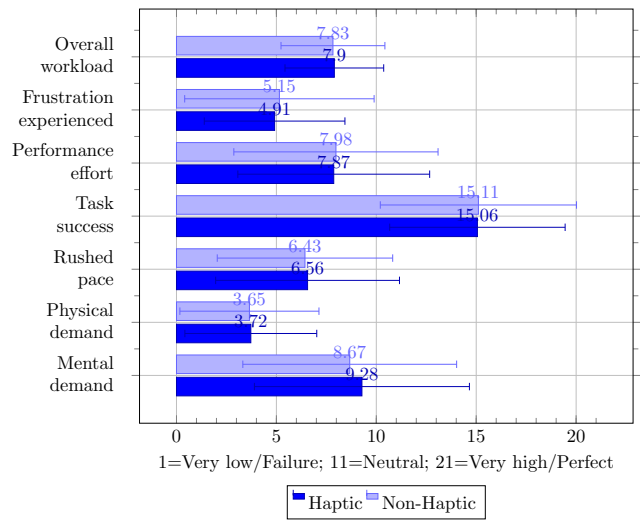

(a) Task Load Index

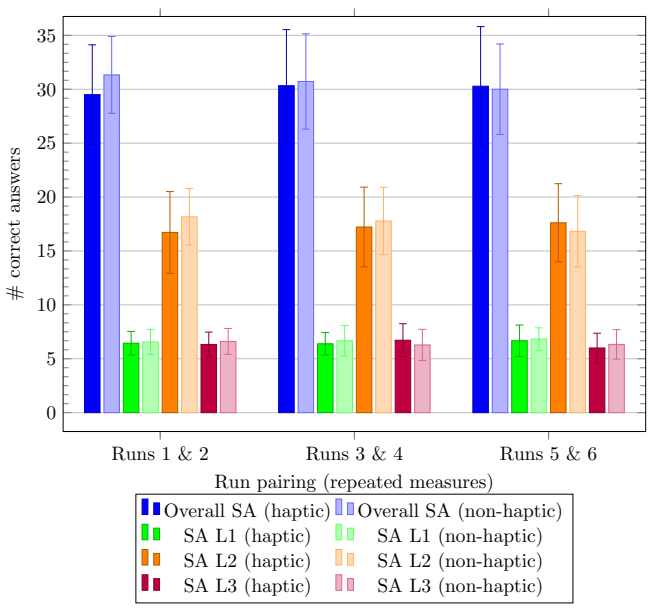

(b) SAGAT

Figure 12 : No significant differences in workload or situation awareness were found between the haptic and non-haptic control modes.

\subsection{Data Collection}

Three questionnaires, the SAGAT technique, and manual event logging were used during the experiment. A pre-experiment questionnaire was used to collect demographic information. Of particular interest were questions about past experience with technology, remote control cars, and video games, as well as participants' recorded attitude toward using technology (Parasuraman, 2000) and taking risks (Mick \& Fournier, 1998). In addition, the ETS Cube Comparison Test (Ekstrom et al., 1979) was administered to evaluate participants' spatial reasoning (SR) skills. During each run, SAGAT screen questions and operator answers were logged. An experimenter present with the robot kept track of wrong turns and collisions made by the robot using an Android app. The app would send this information to the user interface for determining the correct answers to the SAGAT questions. A post-run questionnaire was administered after each use of the robot (including trial runs), and consisted of a performance self-assessment, the Muir trust scale (Muir, 1989), and NASA TLX workload questions (Hart \& Staveland, 1988). After completing the final post-run questionnaire, a post-experiment questionnaire was administered. This final questionnaire examined how participants felt about the two control modes (i.e., haptic and non-haptic) and their ability to use them.

\subsection{Results and Discussion}

A total of 18 people participated in our experiment (12 males and 6 females). The average age was 21 with a standard deviation of 2 years. Of the 18 participants, 15 reported being students, 2 worked in retail, and one did not respond. There were 16 right-hand-dominant participants, 1 left-hand-dominant, and 1 ambidextrous participant.

Although possibly the result of positivity bias, nearly all prior work regarding the haptic control of mobile robots report the introduction of haptic feedback as either positively influencing operator control or being positively received by users. We found no significant difference in participants' reported cognitive overall workload (Wilcoxon matched-pairs signed-ranks test; $p \leq 0.57, n=$ 52 ) or SA (see Fig. 12). However, we did find that despite having exactly the same underlying navigation system, participants reported significantly higher levels of confidence in communicating 


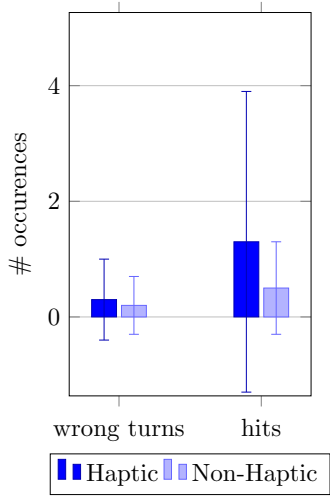

(a) Operator Mistakes

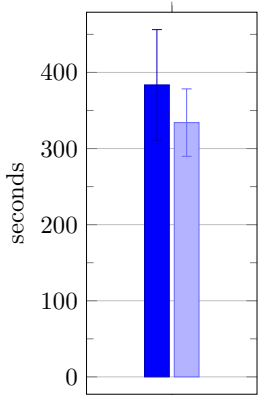

Time to task completion II Haptic $\square$ Non-Haptic

(b) Completion Time

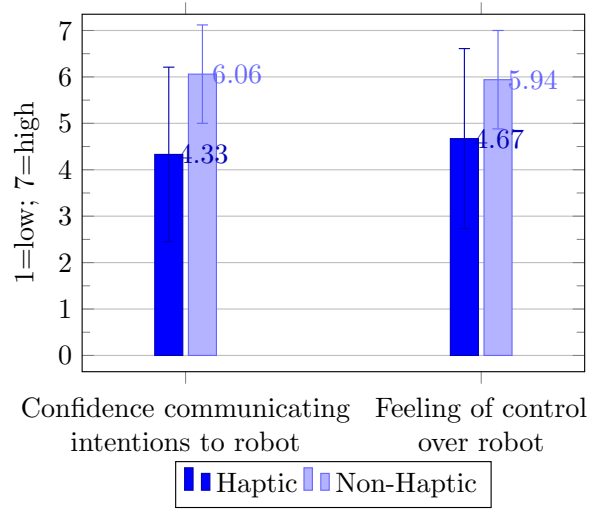

(c) Operator Confidence

Figure 13 : Task Performance and Confidence $(\bar{x} \pm 1 S D)$. (Left) Wrong turns and hits. $\bar{x}$ wrong turn $/$ haptic $=9.3 ; \bar{x}$ wrong turn/non-haptic $=0.2 ; \bar{x}$ hits/haptic $=1.3 ; \bar{x}$ hits $/$ non-haptic $=0.5$ (Center) Time to completion. $\bar{x}$ haptic $=383.6 ; \bar{x}$ non-haptic $=334.1$ (Right) Joystick preference. $\bar{x}$ for confidence/haptic $=4.33 ; \bar{x}$ for confidence/non-haptic $=6.06 ; \bar{x}$ for control $/$ haptic $=4.67 ; \bar{x}$ for control/non-haptic $=5.94$

their intentions to the robot using the non-haptic mode $(p \leq 0.003, t(17)=3.46$ using a twotailed paired t-test with $a=0.05$ ). Unsurprisingly, this meant they also felt more in control using the non-haptic mode $(p \leq 0.024, t(17)=2.47)$ and therefore preferred that mode over the haptic mode, a preference usually established by the second trial run that persisted for the remaining runs $(n=14)$. Comments recorded by the experimenter and in surveys indicate that this may be due to the participants finding it difficult to exercise precise control over the robot while overriding the autonomy in haptic mode. This may be related to the observation that the robot experienced fewer hits in non-haptic mode $(p \leq 0.01, t(107)=2.48)$.

We also observed very different operator interactions with the controls between the two modes. In the non-haptic mode, participants typically pushed the joystick all the way forward and held it against the bezel, causing the robot to drive at full speed, an action normally attributed to lowering the dimensionality of the task of driving. In haptic mode, however, participants tended to let the joystick guide their fingers without exerting any force, sometimes even letting go entirely, despite the fact the robot drove at a much slower rate (the same rate as if the joystick was let go of in non-haptic mode). This resulted in a significantly faster time to completion in non-haptic mode $(p \leq 0.001, t(107)=5.77$ using a two-tailed paired t-test with $a=0.05)$.

To date, this case study is the most direct comparison between haptic and non-haptic control modes of which the authors are aware. Our results highlight the complexity of operator interactions and the difficulty of creating haptic feedback behaviors without side effects.

\section{Examples of Feasible Toolkit Use in Prior Work}

Since the case study in the previous section only investigated one implementation of haptic control, we cannot yet say how it would compare to techniques described in prior work. While the possibility now exists for performing additional experiments to make those comparisons, we would still be unable to compare our re-implemented version of the other control modes with the authors' original version. Had the authors of the following six studies been able to make use of our toolkit, the results 
Brooks, Tsui, Lunderville, and Yanco, Evaluating and Comparing Haptics for HRI

of performing such comparisons would be more meaningful. In this section, we discuss several cases of prior work that we believe could have made use of our toolkit.

\subsection{Operator Performance, Haptic Feedback, and Autonomy (Barnes \& Counsell, 2003)}

This work was centered around improving operator situation awareness, although not explicitly stated as such in the article. They conducted a series of experiments testing five different modes:

- Teleoperation with no haptic feedback,

- Teleoperation with environmental haptic feedback,

- Shared control semiautonomous collision avoidance with no haptic feedback,

- Shared control semiautonomous collision avoidance with environmental haptic feedback, and

- Shared control semiautonomous collision avoidance with behavioral haptic feedback.

Their study already employed a number of our recommendations, including the use of a haptic device well suited to the task (an Immersion Impulse Engine 2000), an experiment that consisted of navigating through a slalom course and measuring time to completion, distance travelled, and number of collisions.

Each of the 10 participants involved in the study performed the driving task once in each mode. The paper does not discuss or imply that any kind of trial runs were used, that the path through the course was changed between runs, or that the order in which participants experienced the different modes was varied between participants. All of these are factors that may point to a learning effect, which in turn would skew results.

Our experiment template could be extended to support each participant running each of the five different trials, multiple times, each over a different map. Although no description of their course was given, it should not be difficult to create a slalom course that meets the requirements outlined by our template, because the experiments were performed in simulation. At the beginning of each session, two trial runs would have been performed to help eliminate learning bias. In addition to the metrics collected (which indirectly indicate relative SA), a pre-run SR test would have been administered, along with post-run workload surveys and subjective questionnaires. The SAGAT technique could have been used to help assess SA. Finally, during runs in which the semiautonomous collision avoidance was used, artificial failures could have been used to introduced what the authors called "extenuating circumstances," which they believed would highlight the advantages of haptic control.

\subsection{Environmental Feedback vs. Environmental + Behavioral Feedback (Lee et al., 2005)}

The authors focused on how different styles of haptic feedback affected operator performance and presence. One of the many notable aspects of this article was the clearly defined theoretical example of a situation in which both environmental feedback in the form of artificial force reflection and behavioral feedback in the form of collision avoidance could be used simultaneously to address shortcomings of using either style independently. Two experiments were carried out and presented. In the first experiment, three methods of feedback were considered:

- No feedback,

- Environmental force feedback, and

- Environmental and behavioral (collision avoidance) combined force feedback.

Twenty participants navigated the robot through a virtual environment. Each participant received three trial runs (one using each method of feedback), and then performed three rounds of trials for a total of nine runs. The trial runs were similar to the real runs but had fewer obstacles. The order in which participants used each method was varied. The number of collisions, time to completion, average speed, average minimum distance to obstacles, collision speeds, and turn rate upon colliding with obstacles were all recorded. At the end of the experiment, participants were asked to fill out a 
questionnaire in which they were asked if they could distinguish between when force feedback was being applied or not, how much the force feedback helped, and if they could distinguish between the different force feedback modes. A second experiment was done in the real world. Conditions were similar to the virtual experiment but had only two possible obstacle settings, because of the limited size of the test environment, and participants only used each mode once.

These experiments used an unmodified SensAble Phantom device for controlling the robot by restricting the movement to a $2 \mathrm{D}$ planar surface, and therefore could have potentially benefited from our joystick modification. Another interesting point is the limitation of physical course variations in the paper. Our recommended experimental setup does not actually involve moving components of the course, which allows for easy adaptation to create as many variations as needed. The increase in number of course variations would have also allowed for each participant to have used each more than once. Additionally, there was concern about not having the robot drive as quickly as possible through the course in order to avoid damaging the hardware. Our recommended course setup can be created using either cardboard or plywood, which can be setup to fall over or shove out of place in the event of a collision.

\subsection{Custom Interface Combining a Steering Wheel With Twin Levers (Nguyen \& Ryu, 2011)}

The authors developed a concept interface that combined the notions of a steering wheel with twinlever differential steering. The goal of their interface was to leverage the general population's familiarity with a steering wheel to increase performance, while also offering lower level independent control of each side of the robot to increase overall mobility. They ran comparative studies between their interface, a Phantom Omni, a Logitech joystick, a gamepad, and a Logitech force feedback steering wheel. For at least one of the studies, a haptic feedback effect described as a "cornering effect" (which could only be implemented on their custom interface) was tested. Participants were asked to steer the robot through a slalom course, and completion times were measured.

While the course setup of their experiments was similar to our recommended practice, the paper does not suggest that the course was modified between trials. Based on the presentation of their results, it appears that each alternative interface was comparatively tested against their proposed interface.

This research also incorporated a Phantom Omni that used only two axis of the device, which could have benefited from our joystick modification. Additional benefits our experiment template has to offer include richer data collection and a reduction of unaccounted-for learning effects.

\subsection{Haptic Wheelchair (Luo et al., 1999)}

This publication describes an experiment in which the authors designed a system that used artificial force reflection to try to help blind people control a powered wheelchair. They used a Microsoft Force Feedback Pro Joystick and used haptic force to prevent users from steering into walls. Two example use cases are discussed in their paper: one in which the system is used to drive around a hallway corner, and another in which a blind operator successfully drives from one room to the next. No experimental analysis was conducted.

This paper is an interesting example of research that could have been performed using our experimental setup. First, rather than remotely operating a robot through the course, the operators themselves would actually be inside the course. While this was not a use case scenario we had originally envisioned, there is no reason why the experimental setup could not be used this way. The second reason this is an interesting example is because the target participant population is visually impaired. Visual cues would normally be used to indicate the path to be followed through the course, which is not applicable for a blind user. Alternatively, verbal instructions could be given after each obstacle has been cleared to instruct users where to go. While most of our suggested measures, 
including workload, SA, performance metrics, and subjective measures would still be applicable, our suggested method of testing SR would not.

\subsection{Haptic Feedback From Motor Current Draw and IMU Values (Mullins et al., 2007)}

The authors used an interesting style of behavioral haptic feedback to indicate the current state of the robot to the operator. The authors provided feedback on the $\mathrm{X}$ axis based on the difference between the rotational velocity of the robot and the commanded value. Feedback on the Y axis was based on motor current draw and inertial measurement units (IMU) readings. In this way, the authors hoped to help operators correct for issues such as over-steering as a result of wheel slippage.

Ten participants took part in two studies in which they were asked to control the robot using an unmodified Phantom Omni device (constrained to two axes). Half were members of the research lab who designed the robot, and the other half were recruited participants who had no prior experience with driving robots. Participants were asked to perform each of two tasks a total of two times, once with non-haptic control and once with haptic control. The first experiment asked participants to hold a robot as close to a specific position as possible on the side of a slippery ramp. The second experiment involved operators driving the robot down an off road course as quickly as possible, with times being recorded and averaged for each user group using each mode.

One of the underlying themes in their research was the emphasis on the robot's use in unstructured environments. It is worth pointing out that although our course was designed to be constructed indoors, there is no particular reason why it could not be setup outside to create the unstructured environment used in this experiment. The experimental design for their second task could have been improved based on our suggested methodology, especially by having each participant perform each task using each mode multiple times (including trial runs) through slightly different conditions, such as those provided by our slalom course. It would also have been interesting to see how the feedback affected operators' SA and workload, as well as subjective measures such as operator preference (rather than time to task completion). Finally, this research group could have also benefited from our joystick modification as the Omni was already constrained to two axes.

\subsection{Artificial Force Reflection With Variable Gain (Farkhatdinov \& Ryu, 2010)}

The authors experimented with the concept of variably adjusting haptic feedback gain as a function of the robot's velocity. The concept was implemented using artificial force reflection and tested using two feasibility studies in simulation and two physical experiments. In one of the experiments, five participants drove a robot down a narrow hallway and around a corner, using an unmodified Phantom Omni haptic device. Time to completion and accuracy were compared using the artificial force reflection with and without the variable gain.

This research would have been able to take advantage of our joystick modification. Additionally, the experiment mentioned above could have been performed using our experiment template, adding an additional performance metric of distance traveled.

However, the second experiment performed revealed a more subtle aspect of their variable feedback gain concept that is not testable using our experiment template. In this second experiment, participants were asked to use the robot to push a box inside markings on the floor. Precise operator movement and intentionally making contact in a controlled manner with an object in the environment was required. As we will discuss in Section 5, our experiment template was not intended nor is suitable for evaluating all use cases, even within the restricted domain of teleoperating and supervising ground-based mobile robots. 
Brooks, Tsui, Lunderville, and Yanco, Evaluating and Comparing Haptics for HRI

\section{Limitations and Future Work}

We have presented our toolkit as a starting point for a more extensive framework in haptic HRI research that can be discussed, revised, and iterated upon by our community. Although the toolkit was designed specifically for the restricted tasks of teleoperation and supervision of ground-based mobile robots, it still has limitations. Most notably, our toolkit does not currently cater to driving tasks that involve pushing or otherwise intentionally colliding with objects in the environment. For example, Farkhatdinov and Ryu (2010) experimented with precision vehicle placement and intentionally contacting objects in a controlled manner by way of pushing a box into a marked area on the floor, demonstrating two important navigation/teleoperation skills that are not covered by our experiment template. Rösch and Schilling (2002) provide an example in which the authors use force reflection (or physical contact) rather than artificial force reflection; that is, the haptic force experienced by the operator is relative to actual force being applied to the robot, in this case, to the front bumper. Another example can be found in Kim, Yun, Park, Choi, and Kim (2004), where physical contact force is used to generate haptic feedback signaling that the vehicle's tracks have good contact with the stairs of a staircase. This introduces yet another aspect of controlling ground-based mobile robots that must be covered-tasks involving inclined surfaces. A second example of inclined maneuvering can be found in Mullins et al. (2007), where the authors performed an experiment in which operators were asked to hold a robot as close to a specific position as possible on the side of a slippery ramp. These are just a few examples of closely related tasks that should be integrated into our toolkit.

Given the need to expand the toolkit to include additional tasks, we believe the next steps that need to be taken are as follows:

- Categorizing the effects, use cases, and tasks that haptic feedback is being used to solve,

- Identifying common metrics that are relevant to all tasks in each category (e.g., Steinfeld et al., 2006), and

- Developing or identifying experiments that best allow us to normalize for differences in hardware-both in the haptic device itself (Hayward \& Astley, 1996) and in the robot (Balakirsky, Carpin, Dimitoglou, \& Balaguer, 2009) — and to look at the relative metrics.

The ability to categorize haptic feedback is a crucial step toward a common framework for evaluating and comparing the use of haptic feedback for HRI. Some types of classification have already begun to emerge that are useful for describing many haptic interfaces and may also prove effective for finding common metrics. Within the category of environmental feedback, feedback behaviors can again be broadly categorized as generating artificial forces (such as artificial force reflection or a forcefield) versus contact/impact forces generated by touching remote objects. Input strategies can also be categorized into "position-position" (scaled absolute positioning) and "position-velocity" modes, which describe whether the position of the input is controlling the position or the velocity of the remote vehicle.

As research fields mature, they develop the ability to compare and replicate results. By developing a large body of comparable work in the domain of haptic feedback control for ground-based mobile robots, we will be able to better understand the effects of this interaction modality on humanrobot interaction. The toolkit presented in this work represents the first steps in this direction.

\section{Acknowledgements}

The authors would like to extend thanks to Eric McCann for his help with the software, Munjal Desai for experimental design, and Jordan Allspaw and Mikhail Medvedev for their help in running experiments. This work was funded in part by an Army Research Office MURI (W911NF-07-10216) and by the National Science Foundation (IIS-0905228 and IIS-1111125). The PHANToM Omni Haptic Device was provided courtesy of SensAble Technologies Inc. 
Brooks, Tsui, Lunderville, and Yanco, Evaluating and Comparing Haptics for HRI

\section{References}

Anderson, R. J., \& Spong, M. W. (1989, May). Bilateral control of teleoperators with time delay. IEEE Transactions on Automatic Control, 34(5), 494-501.

Bae, B., Koo, T., Park, K., \& Kim, Y. (2001). Design and control of a two degree of freedom haptic device for the application of PC video games. In IEEE/RSJ International Conference on Intelligent Robots and Systems (pp. 1738-1743 vol.3). Maui, HI: IEEE.

Balakirsky, S., Carpin, S., Dimitoglou, G., \& Balaguer, B. (2009). From Simulation to Real Robots with Predictable Results: Methods and Examples. In R. Madhavan, E. Tunstel, \& E. Messina (Eds.), Performance Evaluation and Benchmarking of Intelligent Systems (pp. 113-137-27). USA: Springer.

Barnes, D., \& Counsell, M. (2003). Haptic communication for mobile robot operations. Industrial Robot: An International Journal, 30(6), 552-563.

Bowman, D. A., Coquillart, S., Froehlich, B., Hirose, M., Kitamura, Y., Kiyokawa, K., \& Stuerzlinger. (2008). 3D user interfaces: New directions and perspectives. Computer Graphics and Applications, IEEE, 28(6), 20-36.

Brooks, D. J., Lunderville, M., \& Yanco, H. A. (2013). Design of a 2D joystick for robot control based on a 6 DOF haptic device. In Technologies for Practical Robot Applications (TePRA), 2013 IEEE International Conference on (pp. 1-6). Woburn, MA.

Cho, S. K., Jin, H. Z., Lee, J. M., \& Yao, B. (2010). Teleoperation of a mobile robot using a force-reflection joystick with sensing mechanism of rotating magnetic field. IEEE/ASME Transactions on Mechatronics, 15(1), 17-26.

Christensen, Q. M. (2011). Three degree of freedom haptic feedback for assisted driving of holonomic omnidirectional wheelchairs (Unpublished doctoral dissertation). The University of Utah, Salt Lake City, UT.

Desai, M. (2012). Modeling trust to improve human-robot interaction (Unpublished doctoral dissertation). University of Massachusetts Lowell, Lowell, MA.

Desai, M., Kaniarasu, P., Medvedev, M., Steinfeld, A., \& Yanco, H. (2013). Impact of robot failures and feedback on real-time trust. In Proceedings of the 8th ACM/IEEE International Conference on Humanrobot Interaction (pp. 251-258).

Desai, M., Medvedev, M., Vazquez, M., McSheehy, S., Gadea-Omelchenko, S., Bruggeman, C., ... Yanco, H. (2012, March). Effects of changing reliability on trust of robot systems. In Proceedings of the 7th ACM/IEEE International Conference on Human-Robot Interaction (HRI) (p. 73-80).

Diolaiti, N., \& Melchiorri, C. (2003, September). Haptic tele-operation of a mobile robot. In Proceedings of the 7th IFAC Symposium on Robot Control (SYROCO'03) (Vol. 1, p. 7). Wroclaw, Poland.

Ekstrom, R. B., French, J. W., \& Harman, H. H. (1979). Cognitive factors: Their identification and replication. Multivariate Behavioral Research Monographs, 79(2), 3-84.

Endsley, M. (1988, May). Situation awareness global assessment technique (sagat). In Proceedings of the IEEE National Aerospace and Electronics Conference (NAECON) (p. 789-795 vol.3). Dayton, OH. doi: 10.1109/NAECON.1988.195097

Farkhatdinov, I., \& Ryu, J.-H. (2010, Oct). Improving mobile robot bilateral teleoperation by introducing variable force feedback gain. In Proceedings of the IEEE/RSJ International Conference on Intelligent Robots and Systems (IROS) (p. 5812-5817). Taipei. doi:10.1109/IROS.2010.5649112

Hart, S. G. (2006). NASA-task load index (NASA-TLX): 20 years later. In Proceedings of the Human Factors and Ergonomics Society Annual Meeting (Vol. 50, pp. 904-908). doi:10.1177/154193120605000909

Hart, S. G., \& Staveland, L. E. (1988). Development of NASA-TLX (Task Load Index): Results of empirical and theoretical research. Human Mental Workload, 1(3), 139-183.

Hayward, V., \& Astley, O. (1996). Performance measures for haptic interfaces. In G. Giralt \& G. Hirzinger (Eds.), Robotics research (p. 195-206). Springer London. doi:10.1007/978-1-4471-0765-1 22

Hayward, V., Astley, O. R., Cruz-Hernandez, M., Grant, D., \& Robles-De-La-Torre, G. (2004). Haptic interfaces and devices. Sensor Review, 24(1), 16-29.

Kaber, D. B., \& Endsley, M. R. (1997). Out of the loop performance problems and the use of intermediate levels of automation for improved control system functioning and safety. Process Safety Progress, 16(3), 126-131. doi:10.1002/prs.680160304 
Brooks, Tsui, Lunderville, and Yanco, Evaluating and Comparing Haptics for HRI

Kanayama, Y., \& Hartman, B. (1989, May). Smooth local path planning for autonomous vehicles. In Proceedings of the IEEE International Conference on Robotics and Automation (ICRA) (p. 1265-1270 vol.3). Scottsdale, AZ. doi:10.1109/ROBOT.1989.100154

Keyes, B. (2007). Evolution of a Telepresence Robot Interface (Unpublished master's thesis). University of Massachusetts Lowell, Lowell, MA.

Keyes, B., Micire, M., Drury, J. L., \& Yanco, H. A. (2010). Improving human-robot interaction through interface evolution. In D. Chugo (Ed.), Human-robot interaction (chap. 14). InTech. doi:10.5772/8140

Kim, C., Yun, S., Park, K., Choi, C., \& Kim, S. (2004, Sept). Sensing system design and torque analysis of a haptic operated climbing robot. In Proceedings of the IEEE/RSJ International Conference on Intelligent Robots and Systems (IROS) (p. 1845-1848). doi:10.1109/IROS.2004.1389665

Kirkpatrick, A., \& Douglas, S. (2002). Application-based evaluation of haptic interfaces. In Proceedings of the 10th Symposium on Haptic Interfaces for Virtual Environment and Teleoperator Systems (HAPTICS) (p. 32-39). Orlando, FL. doi:10.1109/HAPTIC.2002.998938

Lapointe, J. F., Savard, P., \& Vinson, N. G. (2011, November). A comparative study of four input devices for desktop virtual walkthroughs. Computers in Human Behavior, 27(6), 2186-2191.

Lathan, C. E., \& Tracey, M. (2002). The effects of operator spatial perception and sensory feedback on humanrobot teleoperation performance. Presence: Teleoperators \& Virtual Environments, 11(4), 368-377.

Lee, S., Sukhatme, G., Kim, G. J., \& Park, C.-M. (2005, June). Haptic teleoperation of a mobile robot: A user study. Presence, 14, 345-365.

Lewis, M., Wang, J., \& Hughes, S. (2007, March). USARSim: Simulation for the study of human-robot interaction. Journal of Cognitive Engineering and Decision Making, 1(1), 98-120.

Luo, R., Hu, C.-Y., Chen, T. M., \& Lin, M.-H. (1999). Force reflective feedback control for intelligent wheelchairs. In Proceedings of the IEEE/RSJ International Conference on Intelligent Robots and Systems (IROS) (p. 918-923). Gyeongiu,South Korea. doi:10.1109/IROS.1999.812797

Meijden, O. A. J., \& Schijven, M. P. (2009, January). The value of haptic feedback in conventional and robotassisted minimal invasive surgery and virtual reality training: A current review. Surgical Endoscopy, 23(6), 1180-1190. doi:10.1007/s00464-008-0298-x

Mick, D. G., \& Fournier, S. (1998, September). Paradoxes of technology: consumer cognizance, emotions, and coping strategies. Journal of Consumer Research, 25(2), 123-143.

Mitsou, N., Velanas, S., \& Tzafestas, C. (2006, Sept). Visuo-haptic interface for teleoperation of mobile robot exploration tasks. In Proceedings of the 15th IEEE International Symposium on Robot and Human Interactive Communication (ROMAN) (p. 157-163). Hatfield. doi:10.1109/ROMAN.2006.314411

Muir, B. M. (1989). Operators' trust in and use of automatic controllers in a supervisory process control task. Toronto, Canada: University of Toronto.

Mullins, J., Horan, B., Fielding, M., \& Nahavandi, S. (2007, Sept). A haptically enabled low-cost reconnaissance platform for law enforcement. In Proceedings of the IEEE International Workshop on Safety, Security and Rescue Robotics (SSRR) (p. 1-6). Rome. doi:10.1109/SSRR.2007.4381266

Nguyen, B.-H., \& Ryu, J.-H. (2011, June). Haptic interface for intuitive teleoperation of wheeled and tracked vehicles. In Proceedings of the IEEE World Haptics Conference (WHC) (p. 107-112). Istanbul. doi: 10.1109/WHC.2011.5945470

Niemeyer, G., \& Slotine, J.-J. E. (1991, Jan). Stable adaptive teleoperation. IEEE Journal of Oceanic Engineering, 16(1), 152-162. doi:10.1109/48.64895

Nonpolynomial Labs. (2010). libnifalcon. Retrieved from qdot.github.com/libnifalcon

Novint Technologies Inc. (2013). Novint Falcon. Retrieved from www.novint.com/index.php/ novintfalcon

Okamura, A. M. (2004). Methods for haptic feedback in teleoperated robot-assisted surgery. Industrial Robot: An International Journal, 31(6), 499-508.

Parasuraman, A. (2000, May). Technology Readiness Index (TRI): A multiple-item scale to measure readiness to embrace new technologies. Journal of Service Research, 2(4), 307-320.

Park, J., \& Khatib, O. (2006). A haptic teleoperation approach based on contact force control. The International Journal of Robotics Research, 25(5-6), 575-591. 
Brooks, Tsui, Lunderville, and Yanco, Evaluating and Comparing Haptics for HRI

Quigley, M., Gerkey, B., Conley, K., Faust, J., Foote, T., Leibs, J., ... Ng, A. (2009). ROS: An open-source Robot Operating System. ICRA Workshop on Open Source Software.

Richard, C., Okamura, A. M., \& Cutkosky, M. R. (1997, Nov). Getting a feel for dynamics: Using haptic interface kits for teaching dynamics and controls. In Proceedings of the ASME IMECE 6th Annual Symposium on Haptic Interfaces (pp. 15-21). Dallas, TX.

Rösch, O., \& Schilling, K. (2002). Haptic interfaces for the remote control of mobile robots. Control Engineering Practice, 10, 1309-1313.

Rosenberg, L. B. (1995). Virtual haptic overlays enhance performance in telepresence tasks. Proceedings of SPIE, 2351(Telemanipulator and Telepresence Technologies Symposium, 99), 99-108. doi: $10.1117 / 12.197302$

Ruffaldi, E., Morris, D., Edmunds, T., Barbagli, F., \& Pai, D. (2006, March). Standardized evaluation of haptic rendering systems. In Proceedings of the 14th Symposium on Haptic Interfaces for Virtual Environment and Teleoperator Systems (p. 225-232). doi:10.1109/HAPTIC.2006.1627081

Ruspini, D., Kolarov, K., \& Khatib, O. (1997, Sep). Haptic interaction in virtual environments. In Proceedings of the IEEE/RSJ International Conference on Intelligent Robots and Systems (IROS) (Vol. 1, p. 128-133). Grenoble. doi:10.1109/IROS.1997.649024

Samur, E., Wang, F., Spaelter, U., \& Bleuler, H. (2007, Oct). Generic and systematic evaluation of haptic interfaces based on testbeds. In Proceedings of the IEEE/RSJ International Conference on Intelligent Robots and Systems (IROS) (p. 2113-2119). San Diego, CA. doi:10.1109/IROS.2007.4399522

Satler, M., Avizzano, C., Frisoli, A., Tripicchio, P., \& Bergamasco, M. (2009, Oct). Bilateral teleoperation under time-varying delay using wave variables. In Proceedings of the IEEE/RSJ International Conference on Intelligent Robots and Systems (IROS) (p. 4596-4602). St. Louis, MO. doi: 10.1109/IROS.2009.5354744

Schill, F., Mahony, R., Corke, P., \& Cole, L. (2008). Virtual force feedback teleoperation of the insectbot using optical flow. In Proceedings of the Australasian Conference on Robotics \& Automation. Canberra, Australia.

SensAble Technologies Inc. (2011a). OpenHaptics Toolkit. Retrieved from http: / / www. sensable.com/ products-openhaptics-toolkit.htm

SensAble Technologies Inc. (2011b). PHANToM Omni. Retrieved from http://www. sensable.com/ haptic-phantom-omni.htm

Sharek, D. (2011). A useable, online NASA-TLX tool. Proceedings of the Human Factors and Ergonomics Society Annual Meeting, 55(1), 1375-1379. doi:10.1177/1071181311551286

Sharek, D. (2013). NASA-TLX - playgraph. Retrieved from http: / /www • playgraph.com/nasat lx

Sheridan, T. B., \& Verplank, W. L. (1978, July). Human and computer control of undersea teleoperators (Tech. Rep.). Cambridge, MA: Massachusetts Institute of Technology Man-Machine Systems Lab.

Steinfeld, A., Fong, T., Kaber, D., Lewis, M., Scholtz, J., Schultz, A., \& Goodrich, M. (2006). Common metrics for human-robot interaction. In Proceedings of the 1st ACM SIGCHI/SIGART Conference on HumanRobot Interaction (HRI) (pp. 33-40). New York, NY, USA: ACM. doi:10.1145/1121241.1121249

Takemoto, A., Yano, K., Miyoshi, T., \& Terashima, K. (2004, Sept). Operation assist control system of rotary crane using proposed haptic joystick as man-machine interface. In Proceedings of the 13th IEEE International Workshop on Robot and Human Interactive Communication (ROMAN) (p. 533-538). doi: 10.1109/ROMAN.2004.1374817

Voshell, M., Woods, D. D., \& Phillips, F. (2005). Overcoming the keyhole in human-robot coordination: Simulation and evaluation. In Proceedings of the Human Factors and Ergonomics Society Annual Meeting (Vol. 49, pp. 442-446). doi:10.1177/154193120504900348

Daniel J. Brooks, Department of Computer Science, University of Massachusetts Lowell, Lowell, MA. USA. Email dan@ @ cs.uml.edu; Katherine M. Tsui, Department of Computer Science, University of Massachusetts Lowell, Lowell, MA. USA. Email ktsui@cs.uml.edu; Michael Lunderville, Department of Computer Science, University of Massachusetts Lowell, Lowell, MA. USA. Email mikelunderville@gmail.com; Holly A. Yanco, Department of Computer Science, University of Massachusetts Lowell, Lowell, MA. USA. Email holly@cs.uml.edu 